\title{
Numerical and Experimental Studies of the Elastic Enhancement Factor for 2D Open Systems
}

\author{
M. Ławniczak, M. Bialous, V. Yunko, S. Bauch and L. Sirko \\ Institute of Physics, Polish Academy of Sciences, al. Lotników 32/46, 02-668 Warszawa, Poland
}

\begin{abstract}
We present the results of numerical and experimental studies of the elastic enhancement factor $W$ for microwave rough and rectangular cavities simulating two-dimensional chaotic and partially chaotic quantum billiards in the presence of moderate absorption. We show that for the frequency range $\nu=15.0-18.5 \mathrm{GHz}$, in which the coupling between antennas and the system is strong enough, the values of $W$ for the microwave rough cavity lie below the predictions of random matrix theory and on average above the theoretical results of V. Sokolov and O. Zhirov, Phys. Rev. E 91, 052917 (2015). We also show that for the partially chaotic rectangular billiard the enhancement factor $W$ calculated by applying the Potter-Rosenzweig model with $\kappa=2.8 \pm 0.5$ is close to the experimental one.
\end{abstract}

DOI: 10.12693/APhysPolA.128.974

PACS: 05.45.Mt,03.65.Nk

\section{Introduction}

The elastic enhancement factor, introduced more than 50 years ago by Moldauer [1], has been frequently considered in nuclear physics $[2,3]$ and in other fields [4-6]. An enhancement of the elastic collisions over the inelastic ones in scattering phenomena is the characteristic feature of chaotic systems. The elastic enhancement factor $W_{\beta}$ defined as the ratio of variances of diagonal elements of the two-port scattering matrix $\hat{S}$ to off-diagonal elements of this matrix [4-6], where $\beta=1$ or 2 is the symmetry index for systems with preserved and broken time reversal symmetry (TRS), respectively, is especially interesting because it can be used to study realistic open systems also in the presence of strong absorption.

The properties of the elastic enhancement factor $W_{\beta}$ have been studied in several precisely controllable systems such as microwave cavities [7-10] and networks [1113]. The universality of the elastic enhancement factor $W_{\beta=1}$ has been tested in the wave scattering experiments with microwave cavities simulating chaotic quantum billiards $[8,9]$ in the presence of weak and moderate absorption. Dietz et al. [9] have also studied the universality of $W_{\beta}$ in the case of preserved and partially broken time reversal symmetries. Quite recently an extensive study of the inverse of elastic enhancement factor $\Xi=1 / W_{\beta=1}$, including very low absorption regime, has been published by Yeh et al. [10]. The elastic enhancement factor $W_{\beta}$ in a presence of the moderate and large absorption strength defined as follows: $\gamma=2 \pi \Gamma / \Delta$, where $\Gamma$ is the average resonance width and $\Delta$ is the mean level spacing $[5,6]$, $5 \leq \gamma \leq 54.4$ [11-13] has been also studied for microwave irregular networks [14-17] simulating quantum graphs with preserved and broken time reversal symmetry.

Microscopically, the absorption strength $\gamma=\sum_{c} T_{c}$ can be modeled by means of a very big number of weakly coupled to continuum, parasitic channels "c", where $T_{c}=$ $1-\left|\left\langle S_{c c}\right\rangle\right|^{2}$ and $\left\langle S_{c c}\right\rangle$ stands for the average $S$-matrix [6]. In the paper of Kharkov and Sokolov [4] that deals with the transient from the regular to chaotic internal dy- namics of open systems, controlled by the parameter of chaoticity $\kappa$, the openness $\eta$ is introduced instead of the absorption strength $\gamma$, which is described formally by the same formula. To determine the elastic enhancement factor the two-port measurement is necessary. The coupling of the antennas are quantified by the transmission coefficients $T_{\mathrm{a}}=1-\left|\left\langle S_{\mathrm{aa}}\right\rangle\right|^{2}$, where $\left\langle S_{\mathrm{aa}}\right\rangle$ is an average of the diagonal element of the scattering matrix [18]. Thus $2 \pi \Gamma / \Delta=\sum_{d} T_{d}=\left(T_{\mathrm{a}}+T_{b}+\alpha\right)$ with $\alpha=\sum_{c} T_{c}$ representing the internal absorption of the cavity [9]. The reciprocals of $\Gamma$ and $\Delta$ are, respectively, the time that the incoming particle spends on average inside the system and the Heisenberg time.

The elastic enhancement factor $W_{\beta}$ is defined as $[5,6]$ :

$$
W_{\beta}=\frac{\sqrt{\operatorname{var}\left(S_{\mathrm{aa}}\right) \operatorname{var}\left(S_{\mathrm{bb}}\right)}}{\operatorname{var}\left(S_{\mathrm{ab}}\right)},
$$

where $\operatorname{var}\left(S_{\mathrm{ab}}\right) \equiv\left\langle\left|S_{\mathrm{ab}}\right|^{2}\right\rangle-\left|\left\langle S_{\mathrm{ab}}\right\rangle\right|^{2}$ is the variance of the scattering matrix element $S_{\mathrm{ab}}$ of the two-port scattering matrix

$$
\hat{S}=\left[\begin{array}{cc}
S_{\mathrm{aa}} & S_{\mathrm{ab}} \\
S_{\mathrm{ba}} & S_{\mathrm{bb}}
\end{array}\right]
$$

For small and intermediate values of the absorption $\gamma$ the elastic enhancement factor $W_{\beta}$ might depend both on $\gamma$ and on the coupling to the system [8]. Kharkov and Sokolov [4] showed that the elastic enhancement factor for open systems with the transient from the regular to chaotic internal dynamics depends on the parameter of chaoticity $\kappa$ and the openness $\eta$. However, for large absorption strength $\gamma \gg 1$ the elastic enhancement factor can be approximated by the formula $W_{\beta}=2 / \beta[5,6,8]$.

In this paper we present the results of experimental and numerical studies of the elastic enhancement factor $W_{\beta=1}$ for microwave rough and rectangular cavities performed for the frequency range $\nu=15.0-18.5 \mathrm{GHz}$ and $\nu=16.5-18.5 \mathrm{GHz}$, respectively . The cavities were coupled to the vector network analyzer through the antennas, simulating respectively, chaotic and partially chaotic 
two-dimensional (2D) quantum billiards, with preserved time reversal symmetry $(\beta=1)$, in the presence of moderate absorption. Recently, such a two-channel problem (e.g., an experimental chaotic system with two ports "a" and "b") with internal absorption and time reversal symmetry has been numerically considered by Sokolov and Zhirov [19]. It was shown that for the equivalent channels "a" and "b" with the transmission coefficients $T_{\mathrm{a}}=T_{b}=T, 0 \leq T \leq 1$, the enhancement factor depends both on the transmission coefficient $T$ and internal absorption and can take the values $2 \leq W_{\beta=1} \leq 3$. Thereafter, as we limit our interest to the case of preserved TRS, we will use the abbreviation $W \equiv W_{\beta=1}$.

\section{Microwave cavities simulating quantum billiards}

In the experiment we used a microwave rough cavity and a rectangular cavity to simulate a two dimensional (2D) quantum chaotic billiard and a quantum billiard in a transient region between regular and chaotic dynamics [20], respectively. If the excitation frequency $\nu$ is below $\nu_{\max }=c / 2 d$, where $c$ is the speed of light in the vacuum and $d$ is the height of the cavity, only the transverse magnetic $T M_{0}$ mode can be excited inside the cavity. Below the frequency $\nu_{\max }$ the equivalency between the Helmholtz equation describing microwave cavities and the Schrödinger equation describing the quantum systems [21-35] provides the analogy between microwave cavities and quantum billiards. Absorption of the microwave cavities increases with the frequency but more effectively may be changed by an application of microwave absorbers. In this paper we are interested only in low or moderate absorption, for which $W>2[5,6,8]$ and which, in the used cavities, is observed without any additional absorbers. One should mention that the introduction of one-dimensional microwave networks simulating quantum graphs and two-dimensional microwave cavities simulating quantum billiards increased greatly the number of systems which were used to verify wave effects predicted on the basis of quantum physics [36-42].

\section{Experimental setup}

The two-port measurements of the scattering matrix $\hat{S}$ were performed with the rough semicircular [43] and the rectangular microwave cavities in the frequency window 15.0-18.5 GHz. In Fig. 1 the schemes of the rough (part (a)) and the rectangular (part (b)) cavities are shown. The rough cavity is composed of the two side wall segments and two semicircular plates of an aluminium alloy, which contains more than $90 \%$ of pure aluminium. The semicircular segment (I) is described by the function $r(\theta)=r_{0}+\sum_{i=2}^{M} a_{i} \sin \left(i \theta+\phi_{i}\right)$, where the mean radius $r_{0}=20.0 \mathrm{~cm}, M=20$, and $0 \leq \theta<\pi$ with the phases $\phi_{i}$ uniformly distributed on $[0,2 \bar{\pi}]$. The amplitudes $a_{i}$ are uniformly distributed on $[0.084,0.091] \mathrm{cm}$. The surface roughness of a billiard is characterized by the parameter $\langle k\rangle=\left[\left\langle k^{2}(\theta)\right\rangle_{\theta}\right]^{1 / 2}$, where $k(\theta)=(\mathrm{d} r / \mathrm{d} \theta) / r_{0}$, that determines, among others, the state number $N$ above which

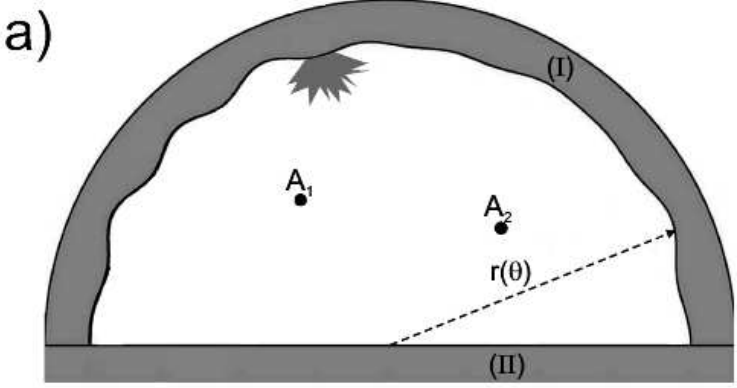

b)

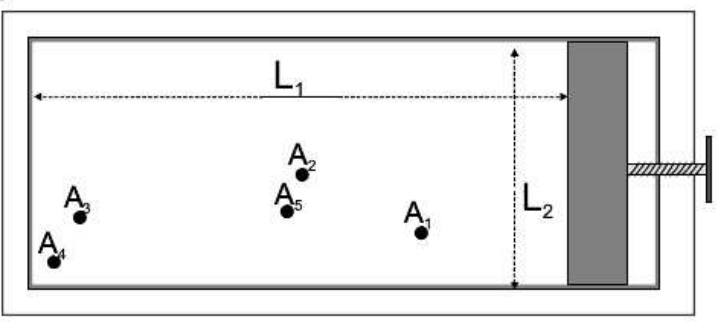

Fig. 1. (a) The rough microwave cavity and (b) the rectangular cavity which were used for measuring the two-port scattering matrix $\hat{S}$. The rough cavity side wall segments are marked by (I) and (II) (see text). The vector network analyzer Agilent E8364B was connected through the flexible microwave cables HP 8513360016 and HP 85133-60017 to the microwave antennas which were introduced inside the cavities (holes $A_{1}, A_{2}$ in panel a) and $A_{1}, A_{2}, A_{3}, A_{4}, A_{5}$ in panel (b)). In order to create different realizations of the rough cavity a metallic perturber (see panel (a)) was moved inside the cavity. The width of the rectangular cavity was $20 \mathrm{~cm}$. The length of the cavity was changed from $L_{1}=41.5$ to $36.5 \mathrm{~cm}$ in 25 steps of $0.2 \mathrm{~cm}$ length.

the cavity is fully chaotic [44-46]. In our case the parameter $\langle k\rangle \approx 0.2[46]$ which defines the limit of chaoticity above $N=208(\approx 10.1 \mathrm{GHz})$. It is important to point out that the presence of the metallic perturber, used to obtain different configurations of the cavity, lowers this limit [20].

The rectangular microwave cavity was made of brass. The width of the cavity was $L_{2}=20 \mathrm{~cm}$ and its length was changed from $L_{1}=41.5$ to $36.5 \mathrm{~cm}$ in 25 steps of $0.2 \mathrm{~cm}$ length. Five holes in the bottom plate of the cavity enable to perform measurements of the scattering matrix $\hat{S}$ for ten different combinations of antennas positions.

All cavities had the same height $d=8 \mathrm{~mm}$, so that $\nu_{\max }=18.7 \mathrm{GHz}$. The wires of microwave antennas (diameter $0.9 \mathrm{~mm}$ ) were protruded $3 \mathrm{~mm}$ into the cavities. The antennas were connected to the vector network analyzer Agilent E8364B through the HP 85133-60016 and HP 85133-60017 flexible microwave cables.

\section{Results}

The averaged transmission coefficient of the $3 \mathrm{~mm}$ long antennas $\bar{T}=\frac{1}{2} \sum_{j=a, b} T_{j}$ with $T_{j}=1-\left|\left\langle S_{j j}\right\rangle\right|^{2}$ as a 
function of the frequency for the both rough and rectangular cavities is shown in Fig. 2 for the whole range of

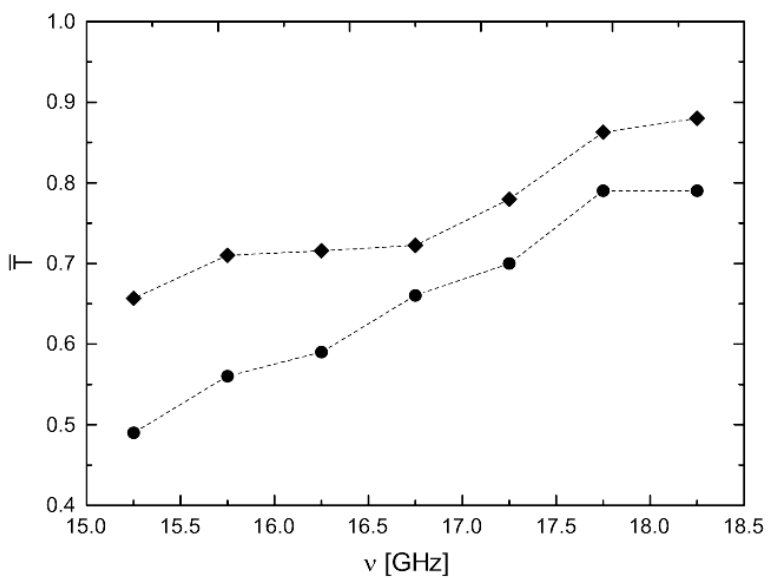

Fig. 2. The averaged transmission coefficient of the $3 \mathrm{~mm}$ long antennas $\bar{T}=\frac{1}{2} \sum_{j=a, b} T_{j}$ with $T_{j}=1-\mid<$ $S_{j j}>\left.\right|^{2}$. Full circles and diamonds mark the averaged transmission coefficients of the rough and rectangular cavities, respectively.

the measurements. The elements of the scattering matrices $\left\langle S_{\text {aa }}\right\rangle$ were averaged over 70 different configurations of the rough cavity in the frequency window $0.5 \mathrm{GHz}$. For the rectangular cavity the elements of the scattering matrix $\left\langle S_{\text {aa }}\right\rangle$ were averaged over 25 lengths of the cavity and ten different combinations of antennas positions in the frequency window $0.5 \mathrm{GHz}$.

In the Table the dependence of the internal absorption $\alpha=\gamma-2 \bar{T}$ of the rough cavity on the frequency $\nu$ is shown in the frequency range $15.0-18.5 \mathrm{GHz}$. The internal absorption of the cavity depends not only on the ohmic losses of the cavity's material but in the reality it is strongly modified by the precision of manufacturing and assembling of the cavity. The total absorption $\gamma$ was obtained by averaging the widths of the well resolved intensity resonances over the frequency window $0.5 \mathrm{GHz}$.

\section{TABLE}

The absorption strength $\gamma$, the averaged transmission coefficient $\bar{T}$ and the internal absorption $\alpha$ of the rough cavity in the frequency range $\delta \nu$.

\begin{tabular}{c|c|c|c}
\hline \hline$\delta \nu[\mathrm{GHz}]$ & $\gamma$ & $\bar{T}$ & $\alpha$ \\
\hline $15.0-15.5$ & 5.02 & 0.49 & 4.04 \\
$15.5-16.0$ & 5.16 & 0.56 & 4.04 \\
$16.0-16.5$ & 5.32 & 0.59 & 4.14 \\
$16.5-17.0$ & 5.82 & 0.66 & 4.50 \\
$17.0-17.5$ & 6.29 & 0.70 & 4.90 \\
$17.5-18.0$ & 6.55 & 0.79 & 4.98 \\
$18.0-18.5$ & 6.82 & 0.79 & 5.24
\end{tabular}

The experimental and numerical results of the elastic enhancement factor $W$ for the rough cavity are shown in Fig. 3. The full circles with the error bars denote the experimental results. The empty circles show the numerical results based on the random matrix theory (RMT) calculated for the total absorption $\gamma$ (see the Table). The triangles stand for the numerical results of Sokolov and Zhirov [19] which were obtained for the two equivalent channels and the internal absorption $\alpha=5.2$. Numerical results of Sokolov and Zhirov are not available for the accurate values of the internal absorption presented in the Table. However, for $4.0<\alpha<5.2$ they should lie slightly above the ones for $\alpha=5.2(\approx 0.03$ for $\nu=15.25 \mathrm{GHz}$ and less for higher frequencies, see [47]). The experimental results for the rough cavity are lying below the RMT predictions and on average above the Sokolov and Zhirov results. Moreover, the experimental results are closer to the Sokolov and Zhirov prediction.

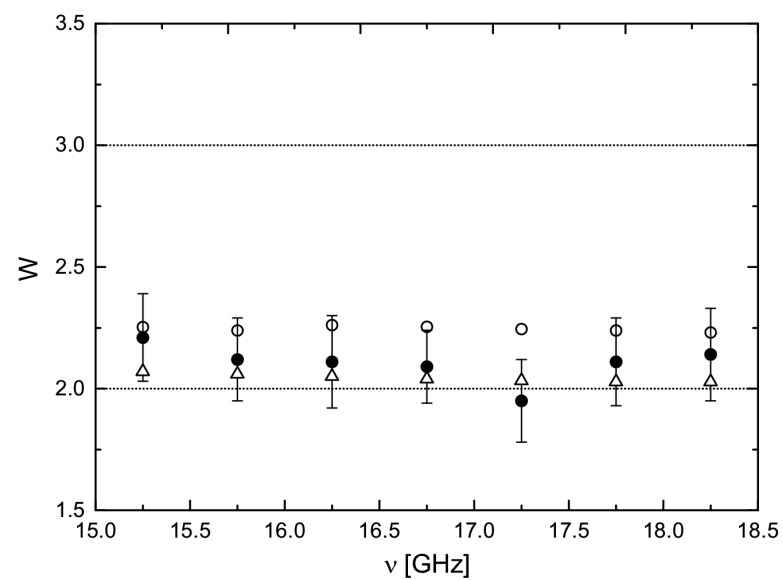

Fig. 3. The elastic enhancement factor $W$ of the rough cavity in the two-channel case. The full circles with error bars denote the experimental results. The empty circles show the RMT results calculated for the total absorption $\gamma$. The triangles show the numerical results obtained for the two equivalent channels and the internal absorption $\alpha=5.2[19]$. The dotted parallel lines $W=2$ and 3 show, respectively, the RMT limits for very strong and very weak absorption.

In Fig. 4 full diamonds show the experimental results obtained for the elastic enhancement factor of the rectangular cavity with the two open channels (see [20] for the details). The experimental results were obtained by averaging of $W$ over 10 different combinations of the positions of the $3 \mathrm{~mm}$ long antennas and over 25 lengths of the cavity in the frequency range $0.5 \mathrm{GHz}$. It was shown in [20] that scattering on microwave antennas moves the system simulated by the rectangular microwave cavity to a transient region between regular and chaotic dynamics. A degree of chaoticity of such a system may be described by a parameter $\kappa$, which changes from $\kappa=0$ for classically integrable systems to $\kappa \rightarrow \infty$ for chaotic systems. In our case $\kappa=2.8 \pm 0.5$ [20]. In such a situation the elastic enhancement factor of the two-port scattering matrix can be expressed by [4]: 


$$
W=3-\gamma \int_{0}^{\infty} \mathrm{d} \tau \mathrm{e}^{-\gamma \tau} b_{2,1}(\tau, \kappa),
$$

where $b_{2,1}(\tau, \kappa)$ is the spectral form factor, which is equal to zero for integrable systems with preserved time reversal symmetry, which immediately leads to $W=3$. On the other hand, in the transition region $0<\kappa<\infty$, its analytic form is unknown.

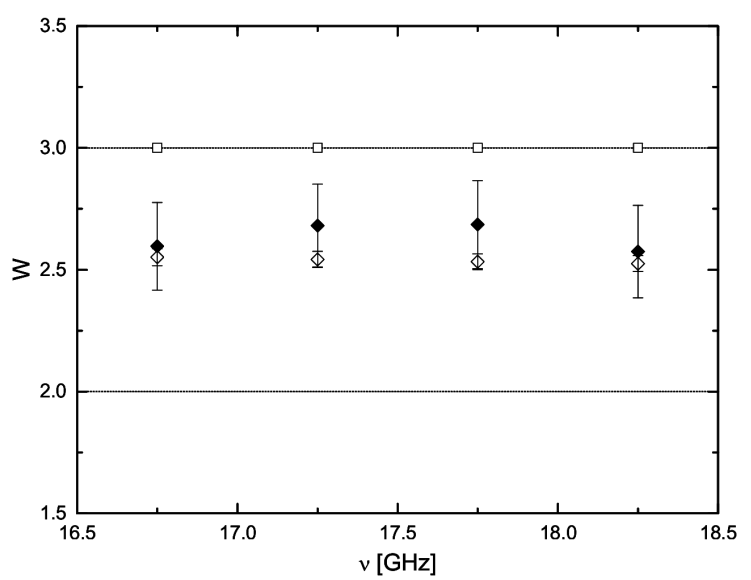

Fig. 4. The elastic enhancement factor $W$ of the twoport scattering matrix $\hat{S}$ of the rectangular cavity (full diamonds) coupled to the two external channels via antennas simulating a quantum system with the chaoticity parameter $\kappa=2.8[20]$. The empty diamonds show the numerical results obtained from the RMT based calculations (formula (3)) by using the spectral form factor $b_{2,1}(\tau, \kappa=2.8)$ given by the formula (4). For comparison, the empty squares show the results of the numerical calculations performed for an integrable system with $b_{2,1}(\tau, \kappa=0)$. The dotted parallel lines $W=2$ and 3 show, respectively, the RMT limits for very strong and very weak absorption. The later one is also expected for the integrable systems.

In order to perform the numerical calculations of the enhancement factor $W$ we used the following formula of the spectral form factor [48]:

$$
\begin{aligned}
& b_{2,1}(\tau, \kappa)=1-K(\tau, \kappa)= \\
& 1-\frac{1}{L}\left|\sum_{l=1}^{L} \mathrm{e}^{\mathrm{i} E_{l} \tau}\right|^{2}-\frac{1}{L}\left|\frac{1-\mathrm{e}^{\mathrm{i} L \Delta \tau}}{1-\mathrm{e}^{\mathrm{i} \Delta \tau}}\right|^{2},
\end{aligned}
$$

where $E_{l}$ are the energy levels of the considered system and $\Delta$ is the mean level spacing equal to 1 after the energy levels unfolding. The energy levels $E_{l}$ were obtained as the eigenvalues of the 500 PotterRosenzweig random matrices [49] of size $5001 \times 5001$ calculated with the transition parameter $\lambda=\kappa / N=$ $2.8 / 5001$ [20]. The rectangular cavity in the frequency range $16.5-18.5 \mathrm{GHz}$ was characterized by the absorption strength, $\gamma=5.6-7.4[20]$.

Figure 4 shows that the enhancement factor $W$ calculated by applying the Potter-Rosenzweig model with $\kappa=2.8 \pm 0.5$ (empty diamonds) is within the experimental errors close to the experimental results (full di- amonds). For comparison, the empty squares show the results of the numerical calculations of $W$ with $\kappa=0$ which yields $W \approx 3$, the limit for the integrable system.

\section{Conclusions}

The elastic enhancement factor $W$ was numerically and experimentally studied for the microwave rough and rectangular cavities simulating two-dimensional chaotic and partially chaotic quantum billiards. The rough cavity in the frequency range $15.0-18.5 \mathrm{GHz}$ was characterized by the moderate absorption strength, $\gamma=5.0-6.8$. We show that the results obtained for the rough cavity lie below the RMT prediction and on average they are above the theoretical results predicted by Sokolov and Zhirov [19]. In the case of a partially chaotic system the elastic enhancement factor $W$ calculated by applying the PotterRosenzweig model with $\kappa=2.8 \pm 0.5$ is within the uncertainties of the experimental results obtained for the rectangular cavity in the frequency range $16.5-18.5 \mathrm{GHz}$. The rectangular cavity was characterized by the absorption strength, $\gamma=5.6-7.4[20]$.

\section{Acknowledgments}

This work was partially supported by the Ministry of Science and Higher Education grants N N202 130239 and UMO-2013/09/D/ST2/03727.

\section{References}

[1] P.A. Moldauer, Phys. Rev. 123, 968 (1961); Phys. Rev. B 135, 642 (1964).

[2] W. Kretschmer, M. Wangler, Phys. Rev. Lett. 41 , 1224 (1978).

[3] J.J.M. Verbaarschot, Ann. Phys. (New York) 168, 368 (1986).

[4] Y. Kharkov, V. Sokolov, Phys. Lett. B 718, 1562 (2013).

[5] Y.V. Fyodorov, D.V. Savin, H.-J. Sommers, J. Phys. A 38, 10731 (2005).

[6] D.V. Savin, Y.V. Fyodorov, H.-J. Sommers, Acta Phys. Pol. A 109, 53 (2006).

[7] C. Fiachetti, B. Michielsen, Electron. Lett. 39, 1713 (2003).

[8] X. Zheng, S. Hemmady, T.M. Antonsen, Jr., S.M. Anlage, E. Ott, Phys. Rev. E 73, 046208 (2006).

[9] B. Dietz, T. Friedrich, H.L. Harney, M. Miski-Oglu, A. Richter, F. Schäfer, H.A. Weidenmüller, Phys. Rev. E 81, 036205 (2010).

[10] J.-H. Yeh, Z. Drikas, J. Gil, S. Hong, B.T. Taddese, E. Ott, T.M. Antonsen, T. Andreadis, S.M. Anlage, Acta Phys. Pol. A 124, 1045 (2013).

[11] M. Ławniczak, S. Bauch, O. Hul, L. Sirko, Phys. Rev. E 81, 046204 (2010).

[12] M. Ławniczak, S. Bauch, O. Hul, L. Sirko, Phys. Scr. T143, 014014 (2011).

[13] M. Ławniczak, S. Bauch, O. Hul, L. Sirko, Phys. Scr. T147, 014018 (2012).

[14] O. Hul, S. Bauch, P. Pakoński, N. Savytskyy, K. Życzkowski, L. Sirko, Phys. Rev. E 69, 056205 (2004). 
[15] M. Ławniczak, O. Hul, S. Bauch, P. Seba, L. Sirko, Phys. Rev. E 77, 056210 (2008).

[16] O. Hul, M. Ławniczak, S. Bauch, A. Sawicki, M. Kuś, L. Sirko, Phys. Rev. Lett. 109, 040402 (2012).

[17] M. Ławniczak, A. Sawicki, S. Bauch, M. Kuś, L. Sirko, Phys. Rev. E 89, 032911 (2014).

[18] R.A. Mendez-Sanchez, U. Kuhl, M. Barth, C.H. Lewenkopf, H.-J. Stöckmann, Phys. Rev. Lett. 91, 174102 (2003).

[19] V.V. Sokolov, O.V. Zhirov, Phys. Rev. E 91, 052917 (2015).

[20] M. Ławniczak, M. Białous, V. Yunko, S. Bauch, L. Sirko, Phys. Rev. E 91, 032925 (2015).

[21] H.J. Stöckmann, J. Stein, Phys. Rev. Lett. 64, 2215 (1990).

[22] S. Sridhar, Phys. Rev. Lett. 67, 785 (1991).

[23] H. Alt, H.-D. Gräf, H.L. Harner, R. Hofferbert, H. Lengeler, A. Richter, P. Schardt, A. Weidenmüller, Phys. Rev. Lett. 74, 62 (1995).

[24] P. So, S.M. Anlage, E. Ott, R.N. Oerter, Phys. Rev. Lett. 74, 2662 (1995).

[25] U. Stoffregen, J. Stein, H.J. Stöckmann, M. Kuś, F. Haake, Phys. Rev. Lett. 74, 2666 (1995).

[26] F. Haake, M. Kus, P. Šeba, H.J. Stöckmann, U. Stoffregen, J. Phys. A 29, 5745 (1996).

[27] L. Sirko, P.M. Koch, R. Blümel, Phys. Rev. Lett. 78, 2940 (1997).

[28] S. Bauch, A. Błędowski, L. Sirko, P.M. Koch R. Blümel, Phys. Rev. E 57, 304 (1998).

[29] L. Sirko, S. Bauch, Y. Hlushchuk, P.M. Koch, R. Blümel, M. Barth, U. Kuhl, H.-J. Stöckmann, Phys. Lett. A 266, 331 (2000).

[30] N. Savytskyy, A. Kohler, S. Bauch, R. Blümel, L. Sirko, Phys. Rev. E 64036211 (2001).

[31] R. Blümel, P.M. Koch, L. Sirko, Found. Phys. 31, 269 (2001)

[32] J. Barthélemy, O. Legrand, F. Mortessagne, Phys. Rev. E 71, 016205 (2005).
[33] S. Hemmady, X. Zheng, J. Hart, T.M. Antonsen, E. Ott, S.M. Anlage, Phys. Rev. E 74, 036213 (2006).

[34] T. Tudorovskiy, U. Kuhl, H.-J. Stöckmann, Neu J. Phys. 12, 123021 (2010).

[35] Ch. Poli, G.A. Luna-Acosta, H.-J. Stöckmann, Phys. Rev. Lett. 108, 174101 (2012).

[36] R. Blümel, A. Buchleitner, R. Graham, L. Sirko, U. Smilansky, H. Walther, Phys. Rev. A 44, 4521 (1991).

[37] M. Bellermann, T. Bergemann, A. Haffmann, P.M. Koch, L. Sirko, Phys. Rev. A 46, 5836 (1992).

[38] L. Sirko, S. Yoakum, A. Haffmans, P.M. Koch, Phys. Rev. A 47, R782 (1993).

[39] L. Sirko, P.M. Koch, Appl. Phys. B 60 (suppl.), S195 (1995).

[40] L. Sirko, A. Haffmans, M.R.W. Bellermann, P.M. Koch, Europhys. Lett. 33, 181 (1996).

[41] L. Sirko, S.A. Zelazny, P.M. Koch, Phys. Rev. Lett. 87, 043002 (2001).

[42] L. Sirko, P.M. Koch, Phys. Rev. Lett. 89, 274101 (2002).

[43] O. Hul, N. Savytskyy, O. Tymoshchuk, S. Bauch, L. Sirko, Phys. Rev. E 72, 066212 (2005).

[44] K.M. Frahm, D.L. Shepelyansky, Phys. Rev. Lett. 79, 1833 (1997).

[45] Y. Hlushchuk, L. Sirko, U. Kuhl, M. Barth, H.J. Stöckmann, Phys. Rev. E 63, 046208 (2001).

[46] N. Savytskyy, O. Hul, L. Sirko, Phys. Rev. E 70 , 056209 (2004).

[47] V.V. Sokolov, O.V. Zhirov, arXiv:1411.6211v2 [nuclth], 12 Dec 2014.

[48] M. Hanke, The form factor in semiclassical theory and analysis, (2006).

[49] F. Leyvraz, J. Quezada, T.H. Seligman, M. Lombardi, Phys. Rev. Lett. 67, 2921 (1991). 